\title{
On the origin of Sérsic profiles of galaxies and Einasto profiles of dark-matter halos
}

\author{
Carlo Nipoti \\ Department of Physics and Astronomy, Bologna University, viale Berti-Pichat 6/2, I-40127 \\ Bologna, Italy \\ email: carlo.nipoti@unibo.it
}

\begin{abstract}
The surface-brightness profiles of galaxies $I(R)$ and the density profiles of darkmatter halos $\rho(r)$ are well represented by the same analytic function, named after either Sérsic, $I \propto e^{-\left(R / R_{*}\right)^{1 / m}}$, or Einasto, $\rho \propto e^{-\left(r / r_{*}\right)^{\alpha}}$, where $R_{*}$ and $r_{*}$ are characteristic radii. Systems with high Sérsic index $m$ (or low Einasto index $\alpha$ ) have steep central profiles and shallow outer profiles, while systems with low $m$ (or high $\alpha$ ) have shallow central profiles and steep profiles in the outskirts. We present the results of idealized numerical experiments which suggest that the origin of these profiles can be traced back to the initial density fluctuation field: high- $\alpha$ (low- $m$ ) systems form in smooth regions via few mergers, while low- $\alpha$ (high- $m$ ) systems form in clumpy regions via several mergers.
\end{abstract}

Keywords. dark matter — galaxies: bulges — galaxies: elliptical and lenticular, cD — galaxies: formation - galaxies: fundamental parameters — galaxies: structure

\section{Einasto and Sérsic profiles}

The surface-brightness profiles of galaxies are successfully described by the Sérsic (1968) law $I(R) \propto e^{-\left(R / R_{*}\right)^{1 / m}}$, where $R_{*}$ is a characteristic projected radius. The same analytic function, written in the form $\rho(r) \propto e^{-\left(r / r_{*}\right)^{\alpha}}$, where $r_{*}$ is a characteristic intrinsic radius, and known as Einasto (1965) profile, represents well the density distribution of dark-matter halos in cosmological $N$-body simulations (Merritt et al. 2005). The Einasto (or Sérsic) function has the property that a single index ( $\alpha$ or $m$ ) determines the distribution at both small and large radii. When $\alpha$ is low (or $m$ is high) the central profile is steep and the outer profile is shallow, while when $\alpha$ is high (or $m$ is low) the central profile is shallow and the outer profile is steep. Cen (2014) has proposed that profiles in which the central and outer slopes are anti-correlated arise naturally in the standard cosmological model with initial density fluctuations represented by a Gaussian random field (GRF). When the fluctuation field is dominated by long-wavelength modes the system forms via a coherent collapse, leading to a profile shallow in the center and steep in the outskirts. When short-wavelength modes dominate, a steep dense central core is initially formed and late infall of substructures produces an extended envelope. Indications in this direction come from cosmological $N$-body simulations: halos with higher values of $\alpha$ are assembled more rapidly than halos with lower values of $\alpha$ (Ludlow et al. 2013). Similarly, binary merging simulations show that the Sérsic index increases while a galaxy is growing via dissipationless mergers (Nipoti et al. 2003; Hilz et al. 2013).

\section{Numerical experiments}

In Nipoti (2015, hereafter N15) we have presented idealized numerical experiments aimed at isolating the effect of the fluctuation power spectrum shape on the density profile 

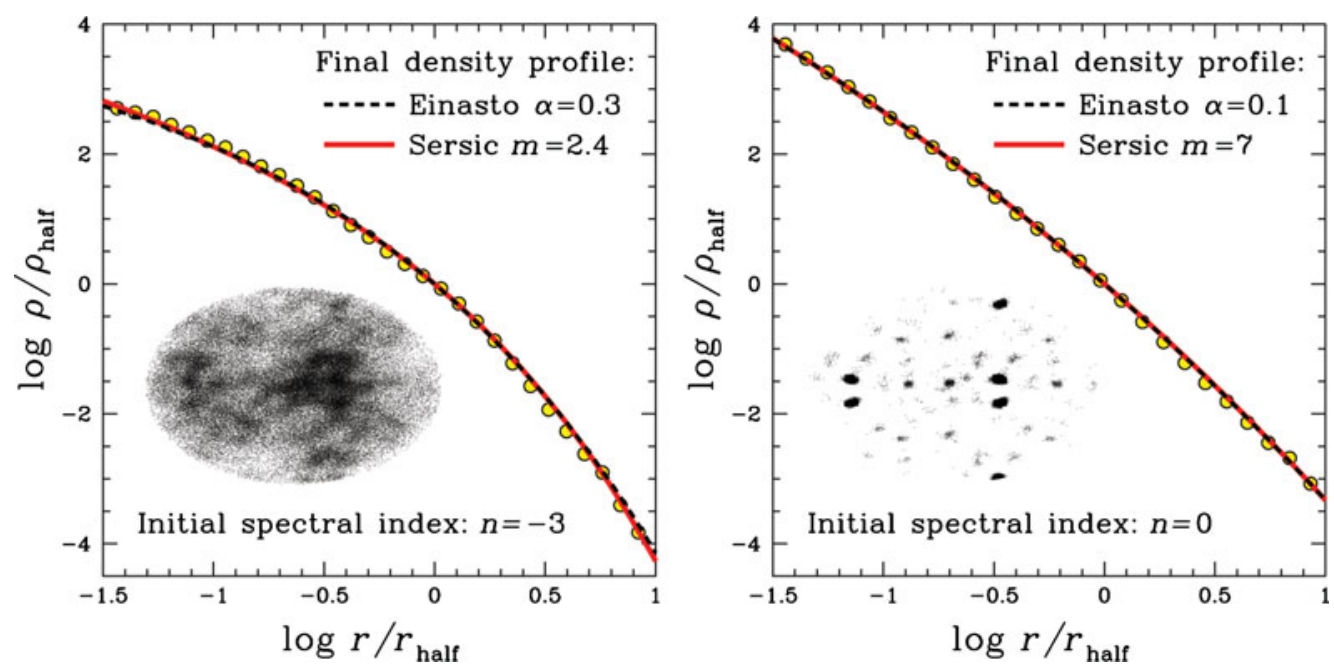

Figure 1. Final angle-averaged density profiles (circles) with their best-fitting Einasto (dashed curves) and deprojected Sérsic (solid curves) profiles for two simulations with initial fluctuation power spectrum index $n=-3$ (left-hand panel) and $n=0$ (right-hand panel; $\rho_{\text {half }}$ is the density at the half-mass radius $\left.r_{\text {half }}\right)$. These two simulations differ from the corresponding simulations presented in N15 only in the specific realization of the GRF. In each panel the initial projected distribution of particles is shown as an inset.

of a system formed via cold dissipationless collapse. We refer the reader to N15 for details of the $N$-body simulations, in which the initial conditions are realized by perturbing a smooth triaxial density distribution with fluctuations generated from a GRF with power spectrum $P(k) \propto k^{n}$, where $k$ is the wave-number. Here we present an additional set of simulations which, for given power-spectrum index $n$, have all the same parameters as the corresponding simulations of N15, but a different realization of the GRF. As done in N15 we fit the final angle-averaged density distributions of the new realizations of the cold collapses with deprojected Sérsic profiles (using the analytic approximation of Lima Neto et al. 1999). In addition we fitted the final angle-averaged density distributions of all simulations (including those of N15) with Einasto profiles, using as an estimate of the half-mass radius $r_{\text {half }}$ the analytic approximation given by Retana-Montenegro et al. (2012). In both cases we have only one free parameter (either $m$ or $\alpha$ ) and we consider the radial range $0.04 \leqslant r / r_{\text {half }} \leqslant 10$. Two examples of these fits are shown in Fig. 1 . The best-fitting values of $\alpha$ and $m$ (and 1- $\sigma$ uncertainties) are reported in Table 1 and plotted in Fig. 2 as functions of the spectral index $n$. We note that the deprojected Sérsic profile, though similar to an Einasto profile, is not exactly an Einasto law, and that the best-fitting $m$ of a deprojected Sérsic is not $1 / \alpha$, where $\alpha$ is the best-fitting Einasto index (Dhar \& Williams 2010). The results of the simulations indicate that $\alpha$ decreases (and $m$ increases) for increasing $n$ : in other words, high- $\alpha$ (low- $m$ ) systems form in smooth regions via few mergers while low- $\alpha$ (high- $m$ ) systems form in clumpy regions via several mergers, in agreement with the model of Cen (2014). Comparing the best-fitting index for two simulations with the same value of $n$ it is apparent that different realizations of the same GRF can lead to significant differences in the final values of $\alpha$ and $m$ (see Fig. 2 and Table 1), consistent with the relatively large scatter in the values of $\alpha$ measured, at fixed halo mass, in cosmological $N$-body simulations (Dutton \& Macciò 2014). 

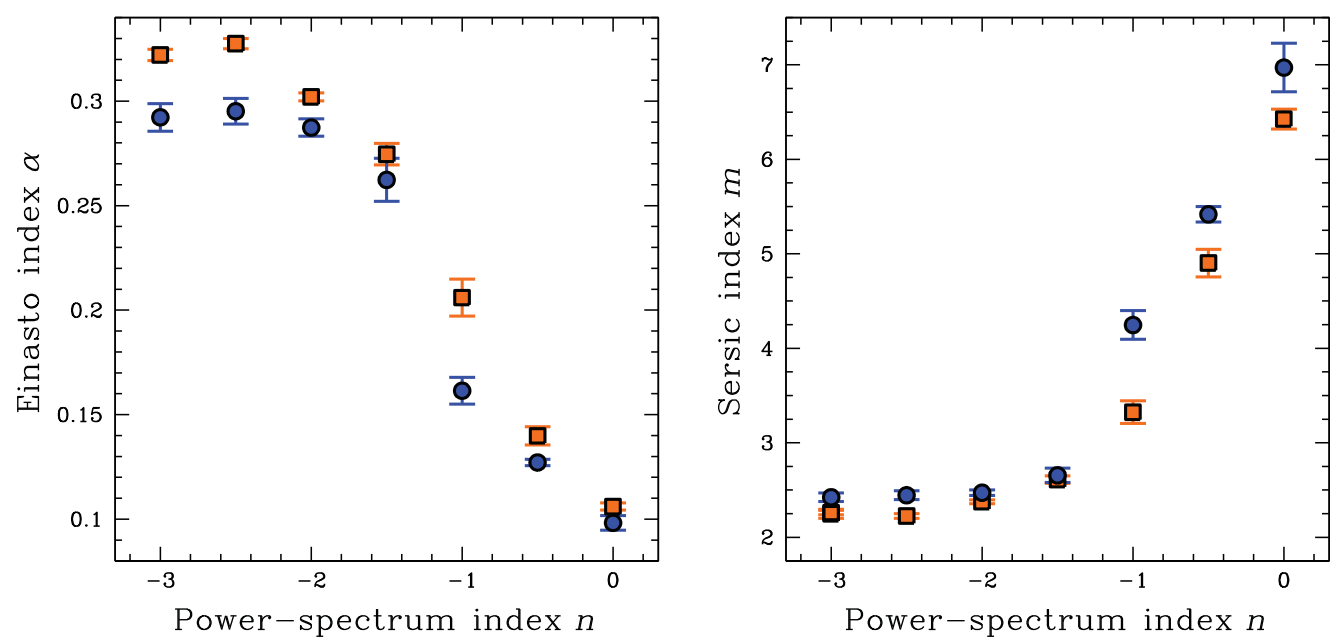

Figure 2. Final best-fitting Einasto index $\alpha$ (left-hand panel) and deprojected Sérsic index $m$ (right-hand panel) as functions of the fluctuation power-spectrum index $n$ of the initial conditions of the $N$-body simulations. Pairs of simulations with the same value of $n$ differ only in the specific realization of the GRF (see Table 1). Error bars indicate 1- $\sigma$ uncertainties.

Table 1. Properties of the simulations. $n$ : power-law index of the initial fluctuation power-spectrum. $\alpha$ and $m$ : best-fitting Einasto and Sérsic indices of the final density profile $( \pm 1-\sigma)$. For each value of $n$ we have two simulations with different realizations of the GRF: (i) N15 (squares in Fig. 2), (ii) this work (circles in Fig. 2).

\begin{tabular}{|r|c|c|c|c|}
\hline$n$ & $\alpha$ (i) & $\alpha$ (ii) & $m$ (i) & $m$ (ii) \\
\hline-3 & $0.322 \pm 0.003$ & $0.292 \pm 0.007$ & $2.26 \pm 0.02$ & $2.42 \pm 0.05$ \\
-2.5 & $0.328 \pm 0.003$ & $0.295 \pm 0.006$ & $2.23 \pm 0.02$ & $2.45 \pm 0.05$ \\
-2 & $0.302 \pm 0.002$ & $0.287 \pm 0.004$ & $2.38 \pm 0.02$ & $2.47 \pm 0.03$ \\
-1.5 & $0.275 \pm 0.005$ & $0.262 \pm 0.010$ & $2.61 \pm 0.04$ & $2.66 \pm 0.08$ \\
-1 & $0.206 \pm 0.009$ & $0.161 \pm 0.006$ & $3.32 \pm 0.12$ & $4.25 \pm 0.15$ \\
-0.5 & $0.140 \pm 0.004$ & $0.127 \pm 0.002$ & $4.90 \pm 0.15$ & $5.42 \pm 0.08$ \\
0 & $0.106 \pm 0.002$ & $0.098 \pm 0.003$ & $6.43 \pm 0.11$ & $6.97 \pm 0.25$ \\
\hline
\end{tabular}

\section{References}

Cen, R. 2014, ApJL, 790, L24

Dhar, B. K. \& Williams, L. L. R. 2010, MNRAS, 405, 340

Dutton, A. A. \& Macciò, A. V. 2014, MNRAS, 441, 3359

Einasto, J. 1965, Trudy Astrofizicheskogo Instituta Alma-Ata, 51, 87

Hilz, M., Naab, T., \& Ostriker, J. P. 2013, MNRAS, 429, 2924

Lima Neto, G. B., Gerbal, D., \& Márquez, I. 1999, MNRAS, 309, 481

Ludlow, A. D., Navarro, J. F., Boylan-Kolchin, M., Bett, P. E., Angulo, R. E., Li, M., White, S. D. M., Frenk, C., \& Springel, V. 2013, MNRAS, 432, 1103

Merritt, D., Navarro, J. F., Ludlow, A., \& Jenkins, A. 2005, ApJL, 624, L85

Nipoti, C., Londrillo, P., \& Ciotti, L. 2003, MNRAS, 342, 501

Nipoti, C. 2015, ApJL, 805, L16

Retana-Montenegro, E., van Hese, E., Gentile, G., Baes, M., \& Frutos-Alfaro, F. 2012, A\&A, 540, A 70

Sérsic, J. L. 1968, Atlas de galaxias australes. Observatorio Astronomico, Cordoba (Argentina) 